\title{
ERRATUM
}

\section{Erratum to: Ankle fracture: radiographic approach according to the Lauge-Hansen classification}

\author{
A. Russo $\cdot$ A. Reginelli $\cdot$ M. Zappia $\cdot$ \\ C. Rossi - G. Fabozzi - M. Cerrato • \\ L. Macarini $\cdot$ F. Coppolino
}

Published online: 1 October 2013

(C) Istituto Ortopedico Rizzoli 2013

Erratum to: Musculoskelet Surg (2013) 97

(Suppl 2):S155-S160

DOI 10.1007/s12306-013-0284-x

In the original article, the coauthor G. Fabozzi name has been published incorrectly. The right name should be G. Fabozzi.

The online version of the original article can be found under doi:10.1007/s12306-013-0284-x.

A. Russo $(\bowtie) \cdot$ G. Fabozzi - M. Cerrato

Department of Radiology, S G. Moscati Hospital, Aversa, Italy e-mail: annarusso81@yahoo.it

A. Reginelli · C. Rossi

Department of Clinical and Experimental Internistic F. Magrassi,

Second University of Naples, Naples, Italy

\section{Zappia}

Department of Health and Science, University of Molise,

Campobasso, Italy

\author{
L. Macarini \\ Department of Radiology, University of Foggia, Foggia, Italy \\ F. Coppolino \\ Department of Radiology, University of Palermo, Palermo, Italy
}

